\title{
Experimental Study on Microseismic Monitoring Highway Bridge Pile Foundation Construction
}

\author{
She Yanhua, Cai Gaojie \\ School of Urban Construction, Yangtze University, Jingzhou, China \\ Email address: \\ syh916@126.com (She Yanhua) \\ To cite this article: \\ She Yanhua, Cai Gaojie. Experimental Study on Microseismic Monitoring Highway Bridge Pile Foundation Construction. American Journal \\ of Civil Engineering. Vol. 7, No. 5, 2019, pp. 141-146. doi: 10.11648/j.ajce.20190705.14
}

Received: November 3, 2019; Accepted: November 21, 2019; Published: December 3, 2019

\begin{abstract}
With the development of urban construction, it is inevitable that new or expanded highways cross or parallel with existing buried pipelines. The impact vibration caused by the construction of perforated pile foundation of highway bridge is bound to have adverse effects on the safe operation of adjacent buried pipelines. Based on the micro seismic monitoring technique, the characteristics of pile foundation construction vibration is researched. A series of sensors are placed on the surface and adjacent pipelines to collect micro seismic events of impact hammer on different hoisting height and different excavation depth of pile foundation construction. Thus, the wave characteristics, spectrum characteristics and energy attenuation law of microseismic signal are analyzed, and the influencing factors of construction vibration are studied. It is found that the peak value of acceleration vibration in the vertical direction is the largest, followed by the peak value of radial acceleration vibration, the vibration energy decays in power relation with the propagation distance, and with the increase of the pile hole depth, the vibration velocity of the same horizontal distance decreases gradually. The attenuation is related to the geological condition of a certain pile hole depth. The research results have some guiding significance for the vibration control of pile foundation construction.
\end{abstract}

Keywords: Pile Foundation Construction, Microseismic Monitoring, Vibration Performance

\section{Introduction}

Impact vibration of highway bridge pile foundation causes deformation of rock and soil medium. This physical deformation will form elastic waves in the stratigraphic structure and propagate in the medium to form seismic waves. The frequency of such "earth pulsation" caused by this excitation is a low-frequency vibration, and the received signal frequency band is lower, generally below $1 \mathrm{kHz}$, and the high frequency components are attenuated during formation propagation, so it is generally called Microseismic [1]. Microseismic is an associated phenomenon in the process of deformation, crack cracking and expansion of rock materials. It is closely related to the mechanical behavior of surrounding rock structure. Therefore, the signal contains a lot of useful information about the stress failure of surrounding rock and the activation process of geological defects.

With the development of geophysics, especially the application of digital seismic monitoring technology, it has provided the necessary technical basis for the study of microseismic in a small area with weak signals. In recent years, scholars from the United States and other countries have carried out a series of tests in Hydropower dam, geothermy, hydraulic fracturing, nuclear waste treatment, oil, mining and other major projects which have successfully demonstrated that microseismic techniques can be applied to those projects [2-4]. At present, China has made different degrees of exploration and application of microseismic technology in oil and gas [5-7], mining [8-11], civil engineering [12-15] and other fields, and gained valuable experience. Due to the extremely complex effect of impact vibration on pipe-soil coupling body, both theoretical analysis and numerical calculation have considerable limitations. Exploring other research methods, especially field measurement method, has been one of the goals pursued by many researchers. In view of the application of microseismic monitoring technology in civil engineering, in this paper, the micro-seismic test of pile foundation construction vibration is carried out, and the micro-seismic monitoring system is applied to the vibration monitoring of bridge pile foundation construction. The test results provide relevant technical guidance for the safety construction of pile foundation in 
adjacent buried pipeline.

\section{Monitoring Instrument Arrangement}

\subsection{Engineering Profile of Monitor Site}

The field microseismic test was conducted at the Juma river no. 8 and no. 9 bridges of the Laiyuan section of Zhangshi expressway across the Shanxi-Beijing pipeline.

No. 8 bridge site is a riverbed terrace area of Juma river. The terrain is slightly undulating, with 9 to $24.5 \mathrm{~m}$ thickness of subsandy soil, Pebble soil and boulder soil accumulated in the riverbed. The underlying Yanshanian complete granite has relatively developed joints and fracture. No. 9 bridge site belongs to the landform of Taihang mountain sub-region, with ' $U$ ' erosion accumulation valleys and erosion terraces. The overburden of the valley in the exposed strata with large fluctuation of the ground is pebble soil and gravel soil with $5 \mathrm{~m}$ thickness. The lithology of bedrock is monzogranite. The rock is relatively hard and complete with developed joints and fractures. There is no bad geological phenomenon in the field area.

\subsection{Instrument Layout and Experimental Process}

According to the topographic condition of the site, three component vibration pickers are arranged at a certain distance from the center of the pile hole. Each place arranged by the vibration picker is called a monitoring station. The station w1 to $\mathrm{w} 4$ receives the vibration response signal, which is recorded and saved by the microseismic acquisition instrument. Vibration displacement and acceleration can be obtained by calibration and conversion of vibration signal.

As shown in Figure 1, it is a schematic diagram of microseismic monitoring station layout at no. 4 pile hole on the left of Juma river no. 8 bridge. The pile foundation is $13.2 \mathrm{~m}$ away from the pipeline. CZ60 percussive hammer is used, with a weight of $4.8 \mathrm{t}$. The specific test of field test is shown in figure 2. The test lasted half a month according to the driving depth of the pile hole. Each test was divided into 5 groups according to the lifting height of the impact hammer of $0.5 \mathrm{~m}$, $1.0 \mathrm{~m}, 1.5 \mathrm{~m}, 2 \mathrm{~m}$ and $2.5 \mathrm{~m}$. The test layout of juma river no. 9 bridge is similar to no. 8 bridge above, as shown as in Figure 2. Field vibration response test was shown as in Figure 3.

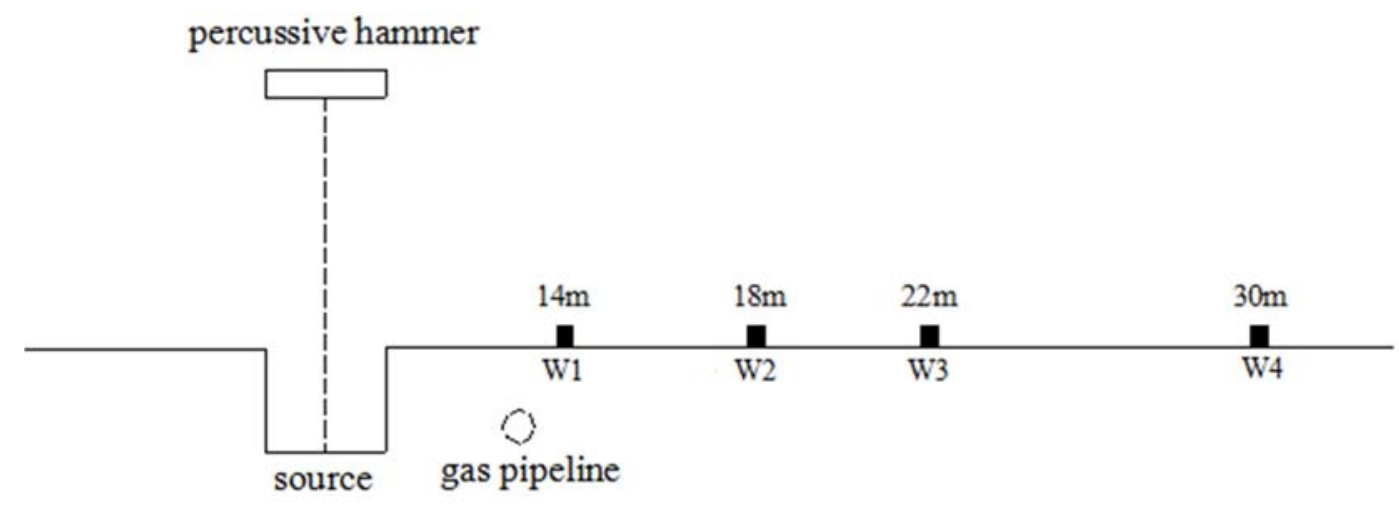

Figure 1. Schematic diagram of monitoring stations allocation.

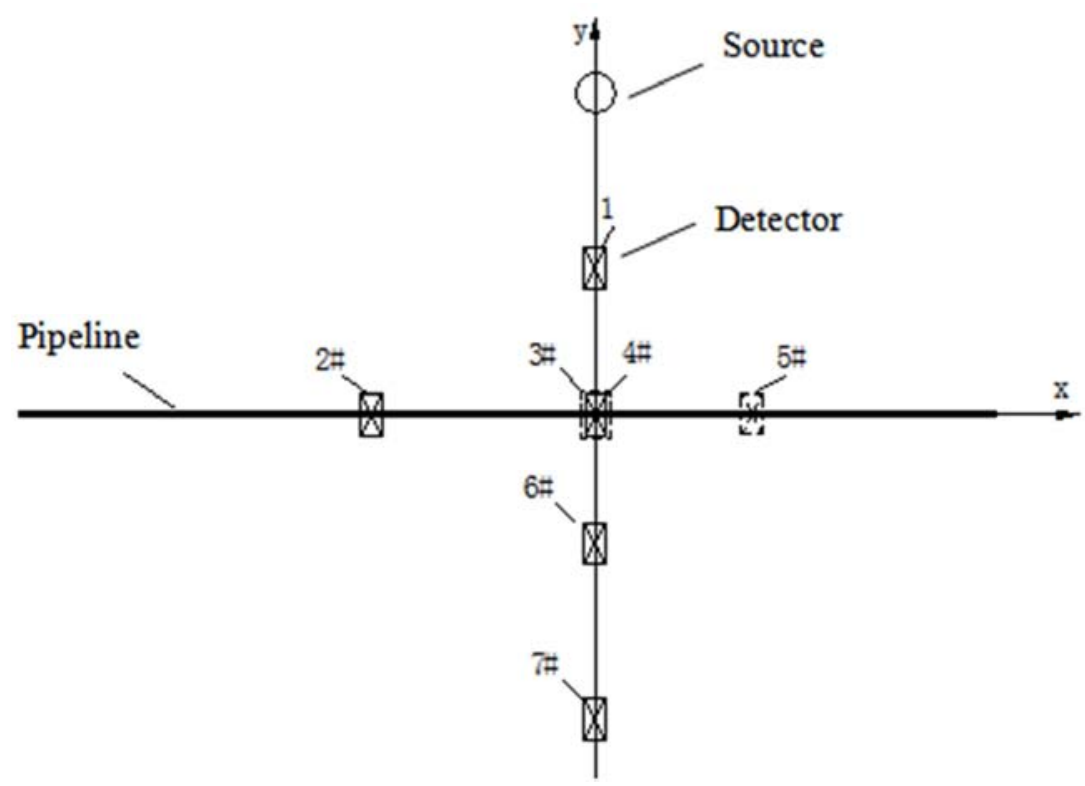

Figure 2. Schematic diagram of test sensors layout. 


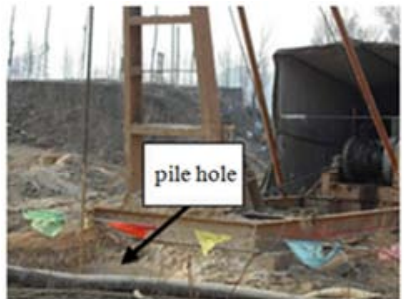

(a) impact vibration source

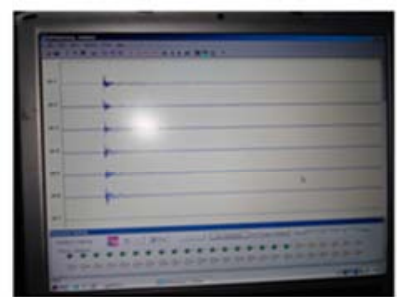

(b) signal collection

Figure 3. Field vibration response test.

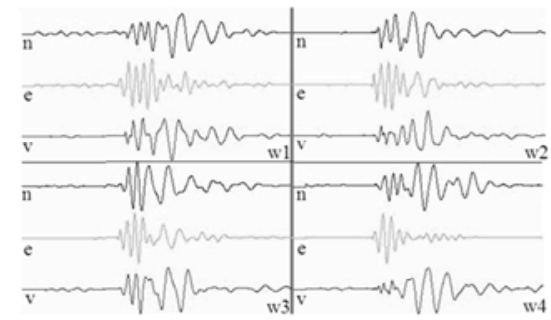

(a) $1 \mathrm{~m}$

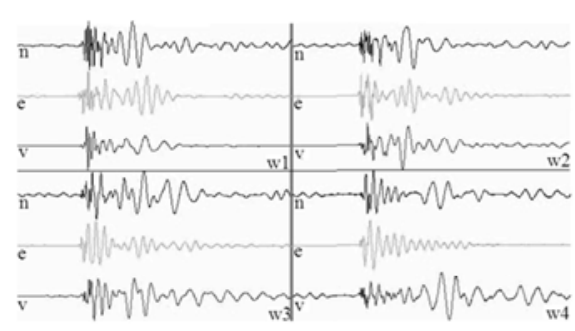

(c) $1 \mathrm{~m}$

\section{Data Processing and Analysis}

\subsection{Typical Waveform of Microseismic Events in Pile Foundation Construction}

The selected typical original signals obtained directly from the test site are shown in Figure 4 and Figure 5, where n, e and $\mathrm{v}$ are the signals measured by the three-component vibration pickup respectively in horizontal radial direction, horizontal tangential direction and vertical direction.

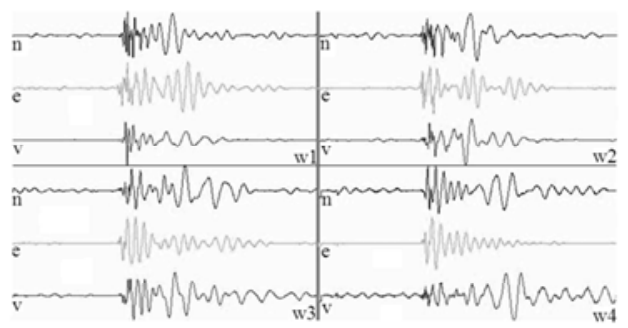

(b) $1.5 \mathrm{~m}$

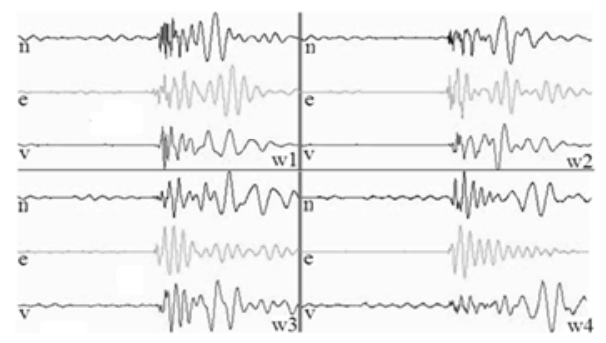

(d) $1.5 \mathrm{~m}$

Figure 4. Typical three component waveforms of microseismic events of hammer different lifting height.

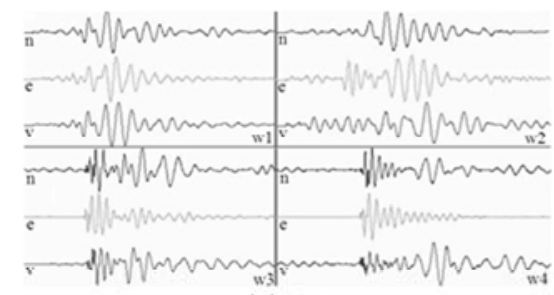

(a) $2 \mathrm{~m}$

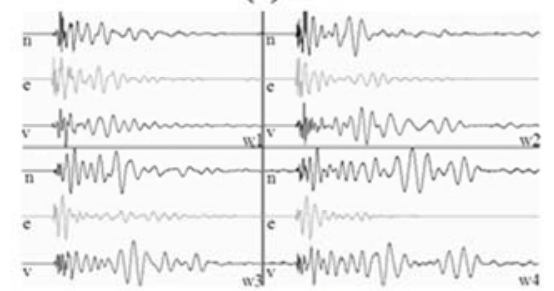

(c) $11 \mathrm{~m}$

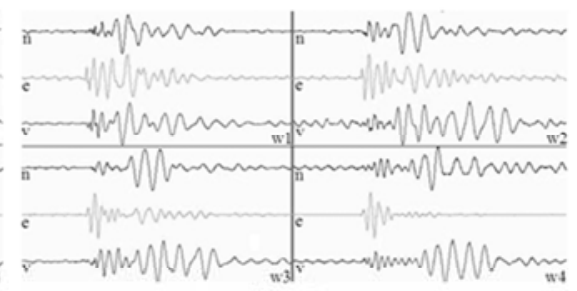

(b) $5 \mathrm{~m}$

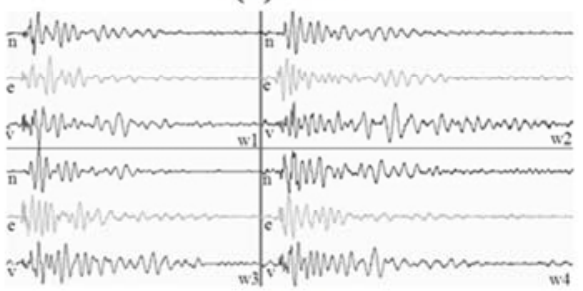

(d) $13 \mathrm{~m}$

Figure 5. Typical three component waveforms of microseismic events at different depth of pile foundation excavation.

It can be seen from Figure 4 and Figure 5 that the waveforms in the three measurement directions are obviously different with the variation of lifting height of the impact hammer and the driving depth of the pile hole. The analysis shows that when the lifting height is $0.5 \mathrm{~m}, 1.0 \mathrm{~m}, 1.5 \mathrm{~m}, 2 \mathrm{~m}$ and $2.5 \mathrm{~m}$, the time between the two adjacent impacts is $3.3 \mathrm{~s}$,
$4 \mathrm{~s}, 5.5 \mathrm{~s}, 6.6 \mathrm{~s}$ and $7.5 \mathrm{~s}$ respectively, but the waveform duration is basically the same, about $0.3 \mathrm{~s}$. In comparison with the common blasting vibration, Figure 6 shows the typical blasting construction vibration waveform. It can be seen that this kind of non-blasting vibration waveform has a long duration and a relatively small vibration frequency. 


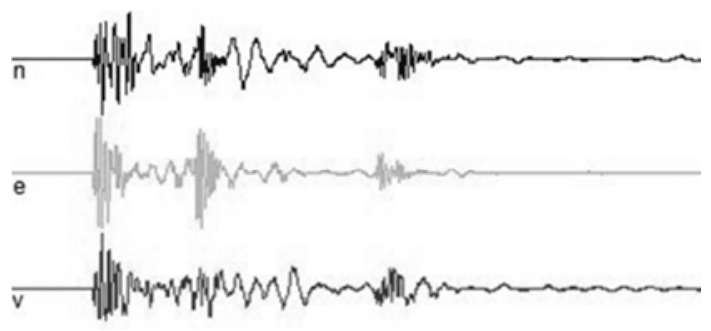

Figure 6. Typical three - component wave of blasting vibration.

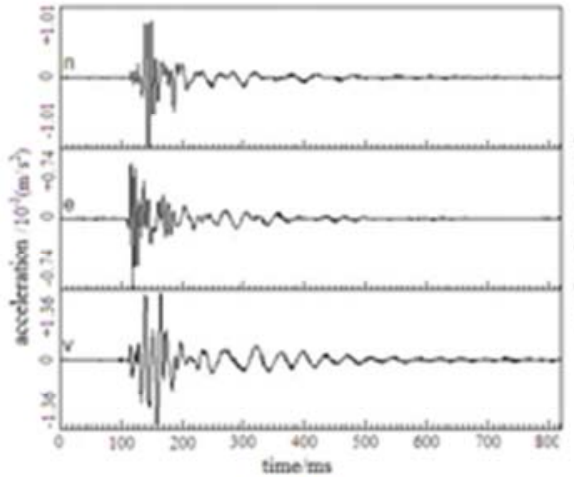

(a) W1

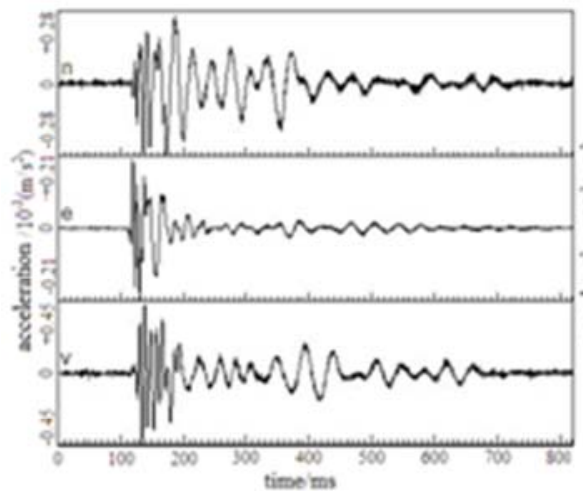

(c) W3

\subsection{Microvibration Response Analysis}

\subsubsection{Vibration Amplitude Analysis}

According to the characteristics of the waveform above, only the time-history curves of acceleration of each station in one of the tests with the lifting height of the impact hammer $2 \mathrm{~m}$ and the driving depth of the pile hole $11 \mathrm{~m}$ are listed, as shown in Figure 7.

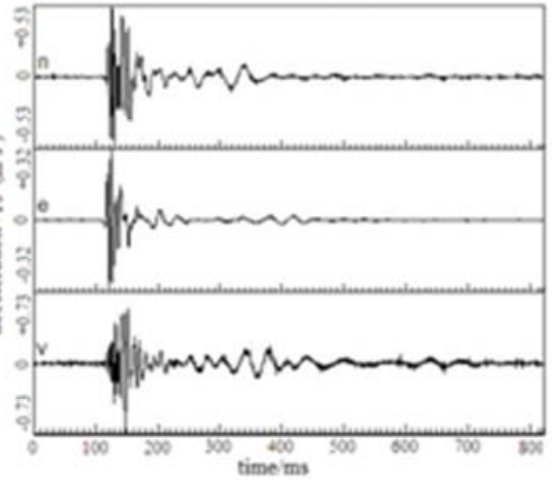

(b) w2

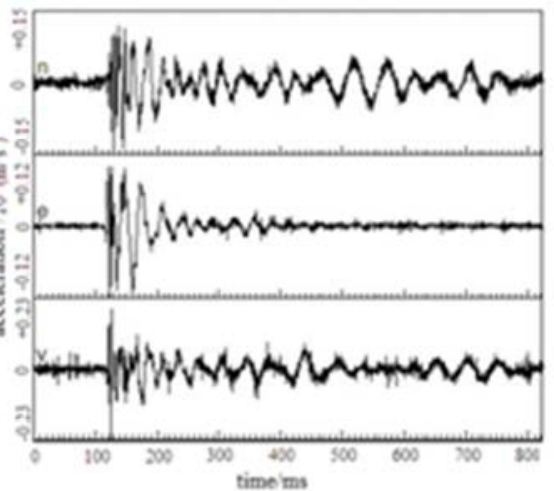

(d) $\mathrm{w} 4$

Figure 7. The acceleration time history curve of each station with a height of $2 \mathrm{~m}$ and a heading depth of $11 \mathrm{~m}$.

It can be seen from Figure 7, complete signals can be collected from three directions of each station. The peak value of acceleration vibration of the medium particle in the vertical direction is the largest, followed by the peak value of radial acceleration vibration, and the influence of tangential vibration can be basically ignored.

\subsubsection{Energy Attenuation Characteristics of Impact \\ Vibration Wave}

The relationship between vibration energy $E$ and vibration acceleration $a$ of each station is as follows [16],

$$
E=10^{3.7849+0.8271 \text { Lna }}
$$

From the above equation, the energy value of impact vibration wave of each station can be calculated, and then the attenuation characteristic curve of impact vibration wave along the propagation distance can be obtained, as shown in Figure 8.

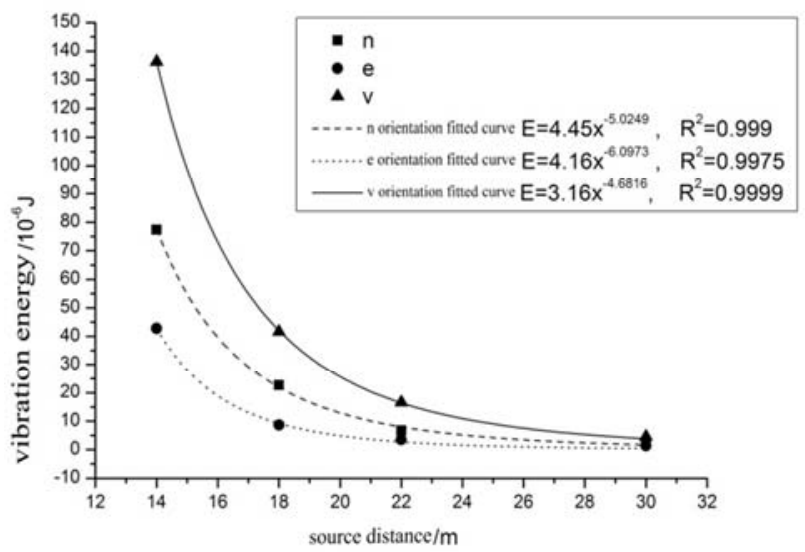

Figure 8. Vibration energy variation curve of each station.

Figure 8 shows that the vertical vibration energy is the main energy, and the vibration energy in the three directions also 
attenuates with the increase of propagation distance. The initial attenuation is very fast, and the attenuation amplitude decreases after a certain distance. It is also known that the energy attenuation index of horizontal direction is larger than that of the other two directions, reaching 6.1.

\subsubsection{Frequency-domain Analysis}

In order to analyze the vibration frequency characteristics of perforated pile construction, typical micro-seismic wave signals were analyzed in the frequency domain, and the power spectrum characteristics of micro-seismic wave in the frequency domain were obtained, as shown in Figure 9. It can be seen from Figure 9, the power of micro-seismic wave signals is mainly concentrated in the frequency range of $10 \mathrm{~Hz}-50 \mathrm{~Hz}$ and has a high signal-to-noise ratio, while the power of vibration wave signals in other frequency bands is very small.

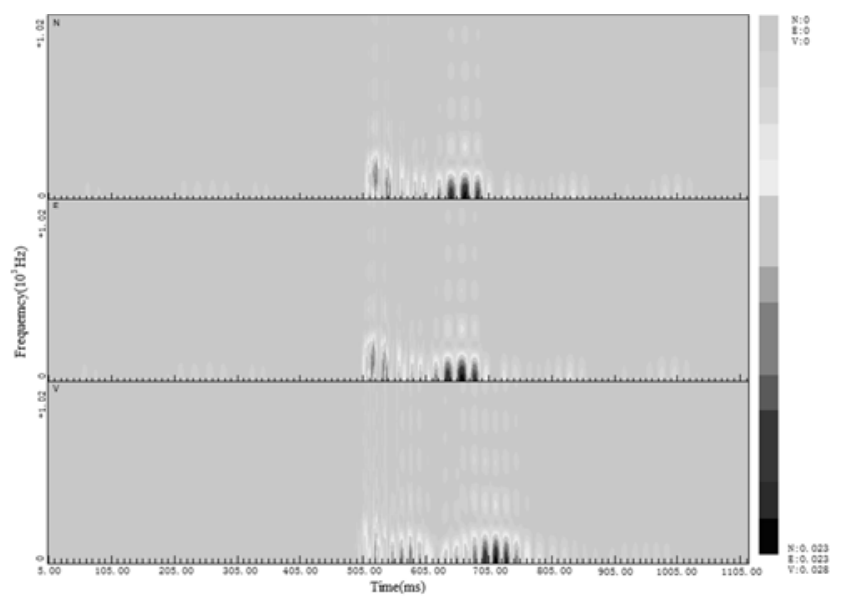

(a) time-frequency

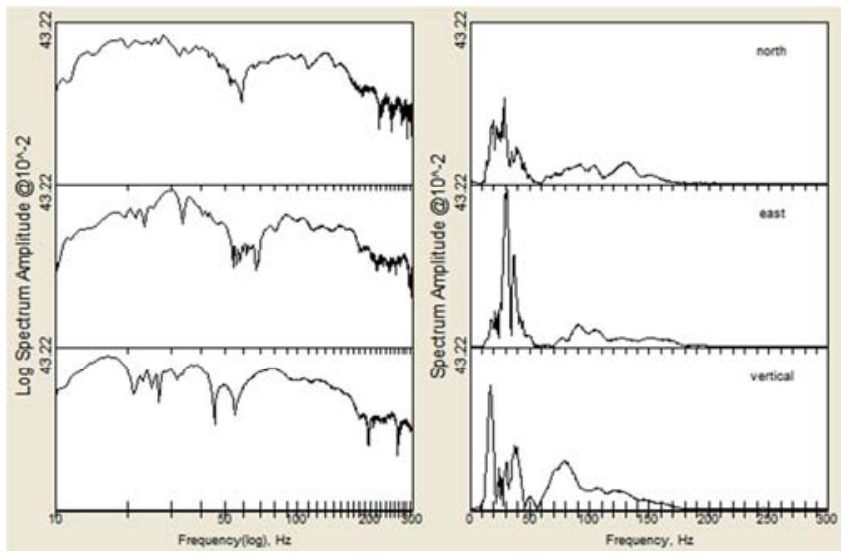

(b) frequency spectrum

Figure 9. The signal frequency analysis.

\subsection{Analysis of Influencing Factors of Construction Vibration}

\subsubsection{The Influence of Percussive Hammer Lifting Height}

In punching pile construction, for the same fixed pile hole, impact energy is the main factor to influence the vibration velocity of each monitoring station, and the impact energy is related to the mass and stroke of the hammer. Figure10 shows the change curve of impact vibration velocity with distance when the hole depth of the test pile is $11 \mathrm{~m}$ and the lifting height changes.

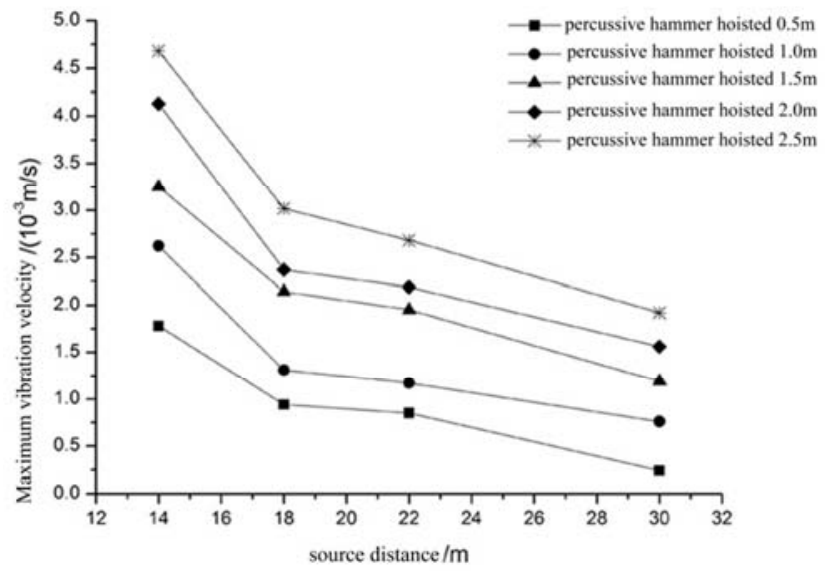

Figure 10. Variation curves of the maximum vibration velocity of hammer different lifting height.

Figure 10 shows that The vibration velocity will increase when percussive hammer height increases. If the construction will cause bad influences to adjacent buildings, percussive hammer height should be lowered while percussive hammer mass is maintained.

\subsubsection{The Influence of Pile Hole Depth}

With the different driving depth of pile hole, the construction vibration velocity will change. Figure 11 shows the vibration velocity of each station at the depth of $2 \mathrm{~m}, 5 \mathrm{~m}$, $8 \mathrm{~m}, 11 \mathrm{~m}, 14 \mathrm{~m}, 17 \mathrm{~m}, 20 \mathrm{~m}$ and $23 \mathrm{~m}$ in the left section of the test pile hole of no. 8 bridge. According to the variation curve of vibration velocity with the depth of pile hole, When the distance to the center of pile foundation is fixed, the vibration velocity will decrease with the increase of pile foundation depth. When the depth is between $11 \mathrm{~m}$ and $14 \mathrm{~m}$, the decaying range is large, probably due to soft soil.

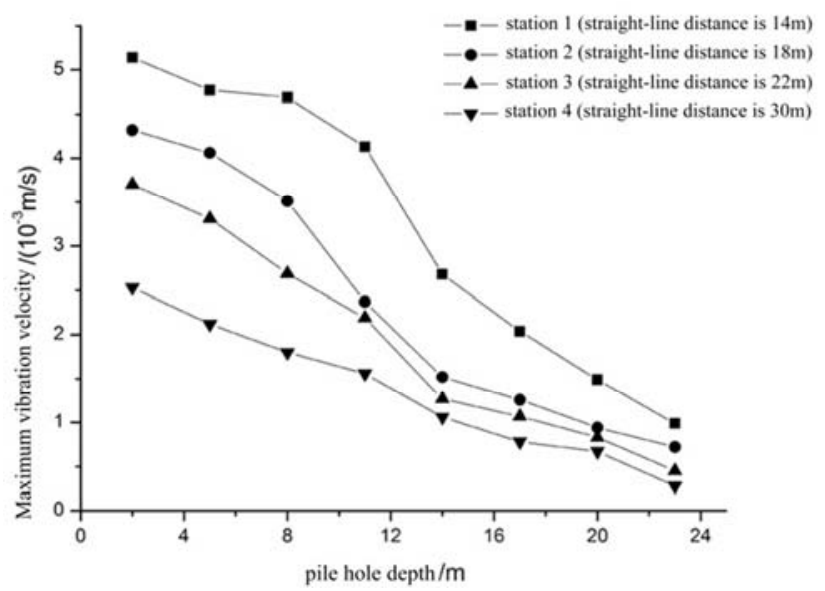

Figure 11. Variation curves of the maximum vibration velocity with the pile foundation depth. 


\section{Conclusion}

In this chapter, the microseismic test of pile foundation construction is carried out. The ground vibration characteristics caused by pile foundation perforated pile construction are studied, and the influence rule of several factors on the ground vibration is analyzed. The microseismic monitoring system can collect complete signals in three directions of each station. The maximum acceleration vibration peak of the medium particle is in the vertical direction, followed by the radial acceleration vibration peak. The regression analysis shows that the vibration acceleration and energy attenuation have the same trend, and they both decline according to the power law correlation with the increase of propagation distance. With the increase of excavating depth, the maximum vibration velocity of the same horizontal distance decreases gradually. The attenuation is related to the geological condition of a certain depth of pile hole.

This practice proves that the micro-seismic monitoring system can better monitor the vibration of pile foundation construction, but in the implementation process, the extraction and processing of micro-seismic signals become the key due to the complex construction environment and a lot of interference signals.

\section{Acknowledgements}

This work is financially supported by National Natural Science Foundation of China (NSFC, 51408057) \& Youth Talent Project of Yangtze University (2015cqr06).

\section{References}

[1] Zhao Xiangdong, Chen Bo, Jiang Fuxing, Chinese Journal of Rock Mechanics and Engineering, Vol. 21 (supplement), 2002, pp. 2612.
[2] Crystal A. Bertoncini, Mark K. Hinders, Measurement, Vol. 43, 2010, pp. 1690-1701.

[3] Lukasz Rudziński, Wojciech Dębski, Journal of Seismology, Vol. 17, 2013, pp. 83-94.

[4] Debotyam Maity, Iraj Salehi, Computers and Geosciences, Vol. 86, 2016, pp. 23-33.

[5] Zuo Lina, Yuan Heping, Liu Zhijuan et al., Well Testing, Vol. 3, 2019, pp. 61-66.

[6] Xi Yan, Li Jun, Zha Chunqing. Journal of Petroleum Science and Engineering, Vol. 180, 2019, pp. 1034-1045.

[7] Li Dewei, Yang Ruizhao, Zhang Du et al., Fault-Block Oil \& Gas Field, Vol. 3, 2019, pp. 346-349.

[8] Zhu Quanjie, Jiang Fuxing, Wei Quande et al., Chinese Journal of Rock Mechanics and Engineering, Vol. 10, 2018, pp. 2319-2333.

[9] Cong Sen, Cheng Jianyuan, Li Xinhu et al., Journal of Xi'an University of Science and Technology, Vol. 4, 2019, pp. 1672-9315.

[10] Liu Fang, Zhao Yanhong, Bao Jinzhe et al., Recent Developments in World Seismology, Vol. 8, 2019, pp. 67-68.

[11] Wang Zhewei, Li Xibin, Shang Xueyi et al., Chinese Journal of Geotechnical Engineering, Vol. 8, 2017, pp. 1408-1415.

[12] Yao Linlin, Zhao Ming, Zhong Guo, Chinese Journal of Underground Space and Engineering, Vol. 5, 2018, pp. 1352-1358.

[13] Yu Qun, Tang Chunan, Li Liancong et al., Journal of Harbin Engineering University, Vol. 11, 2015, pp. 1465-1470.

[14] Gao Zhenping, Li Zhelin, Huang Bo et al., Highway Tunnel, Vol. 3, 2016, pp. 35-40.

[15] Chen Bingrui, Feng Xiating, Zeng Xionghui et al., Chinese Journal of Rock Mechanics and Engineering, Vol. 2, 2011, pp. 275-283.

[16] M. S. Gao, L. M. Dou and N. Zhang: Chinese Journal of Rock Mechanics and engineering, Vol. 26, 2007, pp. 1365. 